\title{
Synthesis and antitumor activities of glucan derivatives
}

\author{
Yuguo Du, ${ }^{a}, *$ Guofeng Gu, ${ }^{a}$ Yuxia Hua, ${ }^{a}$ Guohua Wei, ${ }^{a}$ Xinshan $\mathrm{Ye}^{\mathrm{b}}$ and Guangli $\mathrm{Yu}^{\mathrm{c}}$ \\ ${ }^{a}$ Research Center for Eco-Environmental Sciences, Chinese Academy of Sciences, Beijing 100085, China \\ ${ }^{\mathrm{b}}$ The State Key Laboratory of Natural and Biomimetic Drug, School of Pharmaceutical Science, Peking University, Beijing 100083, China \\ 'Institute of Marine Drug and Food, Ocean University of China, Qingdao 266003, China
}

Received 23 February 2004; revised 12 May 2004; accepted 14 May 2004

Available online 17 June 2004

\begin{abstract}
A highly efficient and practical method for the preparation of $\beta$-D-Glc- $(1 \rightarrow 6)-[\beta-D-G l c-(1 \rightarrow 3)]-\beta-D-G l c-(1 \rightarrow 6)-\beta-D-G l c-(1 \rightarrow 6)-$ $[\beta-D-G l c-(1 \rightarrow 3)]-D-G l c-O M e$ was described. A dendritic nonasaccharide was also synthesized. The antitumor activities of hexasaccharide, the dendrimer, their sulfated derivatives, together with the natural glucan-protein and the corresponding polysaccharide isolated from barmy mycelium of Grifola frondosa, were preliminarily investigated based on Sarcoma-180 studies in mice tests. Our results suggest that the sulfated branching oligosaccharide and natural glycoprotein have better antitumor activities comparing to the parent sugar residue (oligosaccharide or polysaccharide).

(C) 2004 Elsevier Ltd. All rights reserved.
\end{abstract}

\section{Introduction}

A family of glucans containing a main chain of $\beta$-D- $(1 \rightarrow 3)$ glucopyranosyl units, and a short $\beta$-D-glucopyranosyl side chains at O-6 have received considerable attention because of their antitumor activities (immunomodulating action). ${ }^{1}$ Schizophyllan, ${ }^{2}$ scleroglucan, ${ }^{3}$ epiglucan ${ }^{4}$ and lentinan ${ }^{5}$ are the most well-known members of this group of polysaccharides. It is known that the immunopharmacological activities of soluble $(1 \rightarrow 3)-\beta$-D-glucans are closely related to the organization of the $(1 \rightarrow 3)$ - $\beta$-linked backbone into a triple helix, the frequency and the complexity of sidebranching, and their molecular weight. ${ }^{6}$ However, Tsuzuki and co-workers ${ }^{7}$ have also found that the conformation of $\beta$ glucans, either single or triple helix, is independent on the hematopoietic response. To investigate the structureactivity relationship, we have synthesized a series of $\beta$-Dglucosyl oligosaccharides to mimic the repeating units of natural $\beta$-glucan chains. ${ }^{8}$ The mice tests revealed that our previously synthesized $\beta$-D-glucopyranosyl oligosaccharides showing weaker antitumor activities compared to the reported natural polysaccharides. A literature survey suggested that sulfation of the oligosaccharides could result an increasing anti-tumor and anti-HIV activities. ${ }^{9}$ Here, we would like to report the synthesis of sulfated methyl $\beta$-Dglucopyranosyl- $(1 \rightarrow 6)-[\beta$-D-glucopyranosyl- $(1 \rightarrow 3)]-\beta$-Dgluco pyranosyl- $(1 \rightarrow 6)-\beta$-D-glucopyranosyl- $(1 \rightarrow 6)-[\beta-D-$ glucopyranosyl-( $(1 \rightarrow 3)]-\alpha-D-$ glucopyranoside and a sulfated

Keywords: Carbohydrates; Glycosylations; Antitumor agents; Glycodendrimers; Oligosaccharides.

* Corresponding author. Tel.: +86-10-62914475; fax: +86-10-62923563; e-mail address: duyuguo@mail.rcees.ac.cn cluster compound containing three $\beta$-D-glucopyranosyl$(1 \rightarrow 6)$-[ $\beta$-D-glucopyranosyl- $(1 \rightarrow 3)]-\alpha$-D-glucopyranoside components. Interestingly, the hexa- $\beta-D-$ glucoside, $\beta-D-$ Glc- $(1 \rightarrow 6)-[\beta-D-G l c-(1 \rightarrow 3)]-\beta-D-G l c-(1 \rightarrow 6)-\beta-D-G l c-$ $(1 \rightarrow 6)-[\beta-D-G l c-(1 \rightarrow 3)]-D-G l c$, has been well characterized as an elicitor of plant phytoalexin accumulation. ${ }^{10}$ Our research revealed that the sulfated hexa- $\beta$-D-glucoside may also be a potent antitumor agent based on Sarcoma-180 model studies of mice tests.

\section{Results and discussion}

Hexa- $\beta$-D-glucopyranosides (compounds 1 and 2, Fig. 1) have been previously prepared by Takahashi ${ }^{11}$ and Ogawa. ${ }^{12}$ We here modified the synthesis based on our findings of highly efficient and practical synthesis of 3,6branched oligosaccharides. ${ }^{13}$ Thus, phenyl 2,4-di- $O$-acetyl-

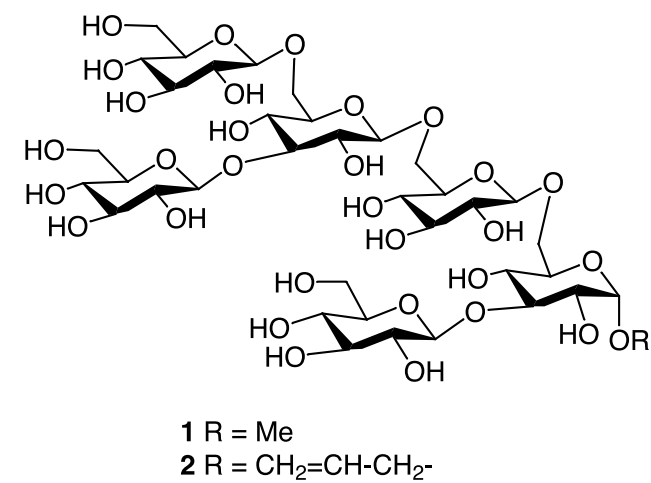

Figure 1. Structures of hexa- $\beta$-D-glucopyranosides $\mathbf{1}$ and $\mathbf{2}$. 

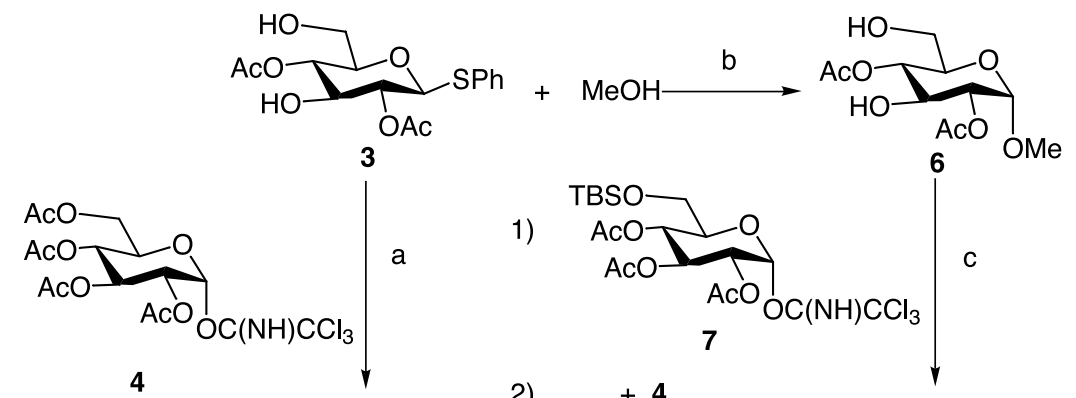

1)
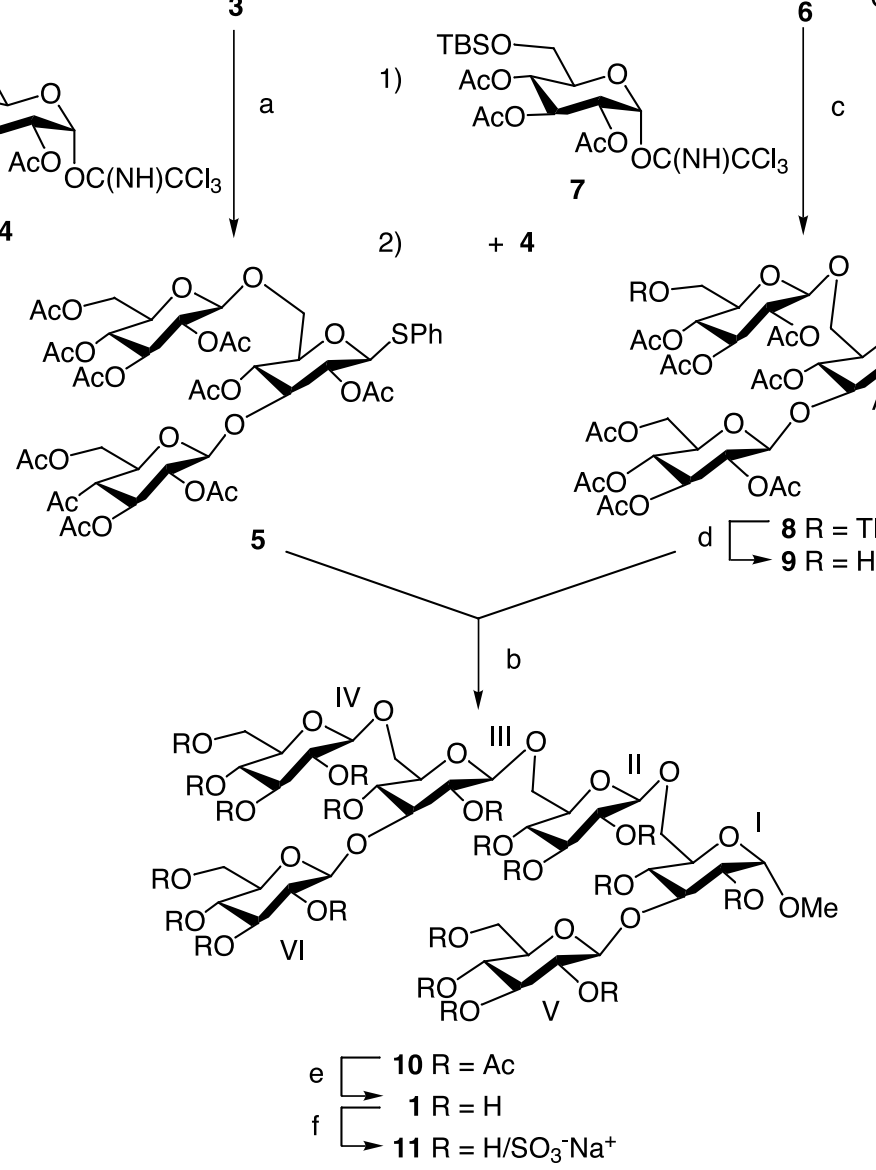

Scheme 1. Synthesis of hexa- $\beta$-D-glucopyranoside 1. Reaction conditions: (a) TMSOTf, $\mathrm{CH}_{2} \mathrm{Cl}_{2}, 0{ }^{\circ} \mathrm{C}, 82 \%$; (b) NIS, TMSOTf, $63 \%$ for $\mathbf{6} ; 86 \%$ for $\mathbf{1 0}$ (from 8); (c) TMSOTf, $\mathrm{CH}_{2} \mathrm{Cl}_{2},-42{ }^{\circ} \mathrm{C}$; then TMSOTf, $0{ }^{\circ} \mathrm{C}, 76 \%$ (two steps); (d) $95 \% \mathrm{TFA}$; (e) $\mathrm{NaOMe}, \mathrm{MeOH}, 93 \%$; (f) $\mathrm{SO}_{3} \cdot \mathrm{Pyr}, \mathrm{DMF}$.

1-thio- $\beta$-D-glucopyranoside $(\mathbf{3})^{13 a}$ was condensed with glycosyl donor 2,3,4,6-tetra- $O$-acetyl- $\alpha$-D-glucopyranosyl trichloroacetimidate $(4)^{14}$ in the presence of trimethylsilyl trifluoromethanesulfonate (TMSOTf) in $\mathrm{CH}_{2} \mathrm{Cl}_{2}$ to give trisaccharide $\mathbf{5}$ in one pot with $82 \%$ isolated yield. Three doublets at $\delta 4.43 \mathrm{ppm}(J=7.9 \mathrm{~Hz}), 4.52 \mathrm{ppm}(J=8.0 \mathrm{~Hz})$ and $4.54 \mathrm{ppm}(J=10.0 \mathrm{~Hz})$ in ${ }^{1} \mathrm{H}$ NMR spectra of 5 clearly indicated all three $\beta$-configuration in this trisaccharide. Thioglycoside $\mathbf{5}$ was used as a latent glycosyl donor in the final assembly of the target hexasaccahride. Attempt to transfer the partially protected donor $\mathbf{3}$ into its methyl glycoside derivative using $N$-iodosuccinimide (NIS) and TMSOTf as catalysts resulted in however $\mathbf{6}$ as a major product $(63 \%)$. The formation of $\alpha$ isomer can be rationalized by a $\mathrm{S}_{\mathrm{N}} 2$ reaction of methanol with 1,6-anhydrosugar intermediate formed from intermolecular ring closure of $\mathbf{3}$ (Scheme 1). ${ }^{15}$

With 3,6-diol 6 in hand, we next applied a one-pot sequential glycosylation to the synthesis of trisaccharide acceptor 9. To this end, 6- $O$-silylated trichloroacetimidate $7^{16}$ (1.1 equiv.) was regioselectively coupled with diol 6 using catalytic amount of TMSOTf (0.07 equiv.) at $-42{ }^{\circ} \mathrm{C}$ in anhydrous methylene chloride. The second donor 4 ( 1.5 equiv.) was added into the above mixture at $0{ }^{\circ} \mathrm{C} 2 \mathrm{~h}$ later, affording trisaccharide $\mathbf{8}$ in $76 \%$ yield within another $2 \mathrm{~h}$. It is noteworthy that an extra amount of TMSOTf (0.01 equiv.) was needed to complete the reaction after the addition of 4. The treatment of $\mathbf{8}$ with $95 \%$ trifluoroacetic acid (TFA) for $1 \mathrm{~h}$ gave trisaccharide acceptor 9. The resulting crude product was co-evaporated with toluene three times and then directly used for the next step without further purification. Coupling of 5 and $\mathbf{9}$ in $\mathrm{CH}_{2} \mathrm{Cl}_{2}$ at $0{ }^{\circ} \mathrm{C}$ under promotion of NIS and TMSOTf gave hexasaccharide 10 in $86 \%$ yield over two steps. ${ }^{1} \mathrm{H}-{ }^{1} \mathrm{H}$ COSY, TOCSY, HMBC and HMQC spectra analyses clearly indicated 6 $\mathrm{H}-1 \mathrm{~s}\left[\delta_{\mathrm{H}} 4.29\left(\mathrm{H}-1^{\mathrm{III}}\right), 4.49\left(\mathrm{H}-1^{\mathrm{II}}\right), 4.51\left(\mathrm{H}-1^{\mathrm{IV}}\right), 4.58\right.$ $\left.\left(\mathrm{H}-1^{\mathrm{VI}}\right), 4.61\left(\mathrm{H}-1^{\mathrm{V}}\right), 4.77\left(\mathrm{H}-1^{\mathrm{I}}\right) \mathrm{ppm}\right]$ and $6 \mathrm{C}-1 \mathrm{~s}\left[\delta_{\mathrm{C}} 96.4\right.$ $\left(\mathrm{C}-1^{\mathrm{I}}\right), 100.6\left(\mathrm{C}-1^{\mathrm{III}}, \mathrm{C}-1^{\mathrm{V}}\right), 100.8\left(\mathrm{C}-1^{\mathrm{VI}}, \mathrm{C}-1^{\mathrm{IV}}\right), 100.9$ $\left.\left(\mathrm{C}-1^{\mathrm{II}}\right) \mathrm{ppm}\right]$, confirming the correct linkages of $\mathbf{1 0}$. Standard Zemplén deacetylation ${ }^{17}$ of $\mathbf{1 0}$ furnished hexa- $\beta$ D-glucopyranoside $\mathbf{1}$ as an amorphous solid. Sulfation of $\mathbf{1}$ with $\mathrm{SO}_{3} \cdot \operatorname{Pyr}$ (10 equiv.) at $50{ }^{\circ} \mathrm{C}$ in $\mathrm{N}, \mathrm{N}$-dimethylformamide (DMF) for 3 days, followed by conversion to the sodium salt, removal of pyridine and purification on a Sephadex LH-20 column, furnished a mixture of sulfated 11. The microanalysis for $\mathbf{1 1}$ was $\mathrm{C} 16.22 \%, \mathrm{H} 1.73 \%$ and $\mathrm{S}$ $19.90 \%$. This highly sulfated mixture was thus obtained in 5 steps at $38 \%$ overall yield starting from $\mathbf{3}$, and was directly used for the following bioassay. 

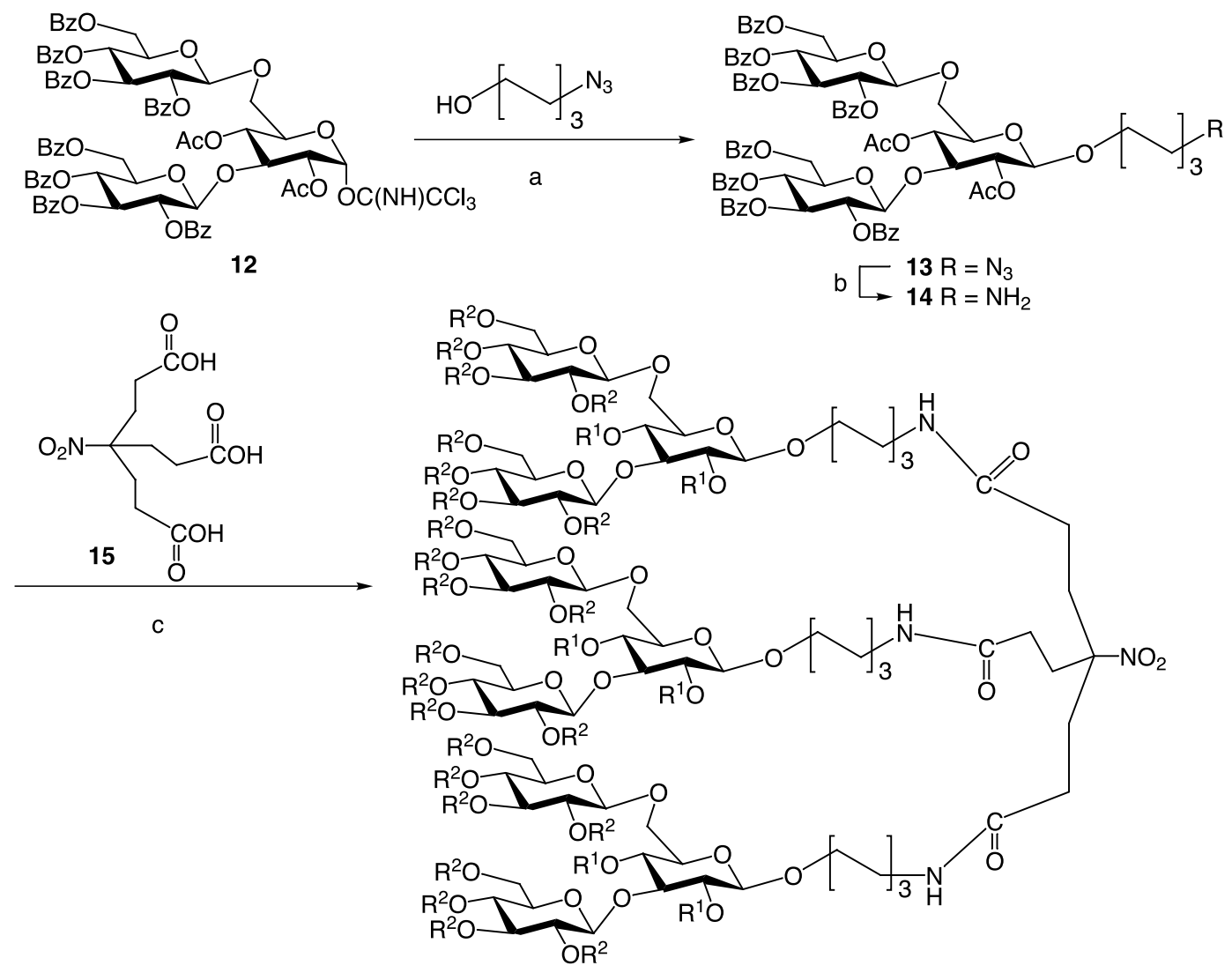

$$
\begin{aligned}
& \text { d }-16 R^{1}=A c, R^{2}=B z \\
& \mathrm{e} \longrightarrow 17 \mathrm{R}^{1}=\mathrm{R}^{2}=\mathrm{H}
\end{aligned}
$$

Scheme 2. Synthesis of nonasaccharide dendritic compound 17. Reaction conditions: (a) TMSOTf, $\mathrm{CH}_{2} \mathrm{Cl}_{2}, 0{ }^{\circ} \mathrm{C}, 84.7 \%$; (b) $\mathrm{Pd}(\mathrm{OH})_{2} / \mathrm{C}, \mathrm{H}_{2}, \mathrm{EtOAc}-\mathrm{EtOH}$, 93.4\%; (c) HOBt, DCC, DMF, rt, 57.2\%; (d) NaOMe, $\mathrm{MeOH}, 91.5 \%$; (e) $\mathrm{SO}_{3} \cdot \mathrm{Pyr}, \mathrm{DMF}$.

Glycodendrimers have been prepared to give rise of new kinds of glycoconjugate derivatives and polysaccharide mimics. ${ }^{18}$ Some of them have shown highly improved bioactivities compared to the monomers. ${ }^{19}$ Encouraged by these results, we prepared a carbohydrate dendrimer based on a combination of 3,6-branched trisaccharides as dendritic components and noncarbohydrate units as trivalent cores (Scheme 2). Thus, the coupling of trisaccharide imidate $\mathbf{1 2}^{\mathbf{8 c}}$ and 6-azido-1-hexanol under standard glycosylation conditions gave 6-azidohexyl 2,3,4,6-tetra- $O$-benzoyl- $\beta$-Dglucopyranosyl- $(1 \rightarrow 6)-[2,3,4,6$-tetra- $O$-benzoyl- $\beta$-D-glucopyranosyl-( $1 \rightarrow 3)]$-2,4-di- $O$-acetyl- $\beta$-D-glucopyranoside (13), a key component for our target synthesis, in high yield. $\mathrm{Pd}(\mathrm{OH})_{2}$ catalyzed hydrogenation of $\mathbf{1 3}$ gave amine derivative 14, which was further condensed with triacid $15^{20}$ in the presence of HOBt and DCC in DMF to give fully protected trimer $\mathbf{1 6}$ in $57.2 \%$ yield. The newly formed amide bond was characterized by $\mathrm{CON} H$ peaks appearing at $\delta 6.04 \mathrm{ppm}(3 \mathrm{H}, J=5.5 \mathrm{~Hz})$ in ${ }^{1} \mathrm{H}$ NMR spectrum, and further confirmed with a mass of $4804(\mathrm{M}+\mathrm{Na})^{+}$of MALDITOF-MS spectrum. Deacylation of $\mathbf{1 6}$ with $1 \mathrm{~N}$ $\mathrm{NaOMe}$ afforded free nonasaccharide dendritic compound 17. Further sulfation of $\mathbf{1 7}$ with $\mathrm{SO}_{3} \cdot \operatorname{Pyr}$ (10 equiv.) in DMF as described in the preparation of $\mathbf{1 1}$, furnished the desired dendrimer 18. Sodium salt of 18, after purification on LH-20 column, was directly used for the next bioassay.
Working for the same project to investigate possible antitumor $\beta$-glucan, we have also extracted and isolated a glucan protein (19) from barmy mycelium of Grifola frondosa (Maitake) with a molecular weight of $95 \mathrm{~K}$. After removal of the protein (accounts for 24\% of total molecular weight), a pure polysaccharide (20) was obtained. The structure of this polysaccharide is determined as a $\beta$-Dglucan with the following basic repeating unit (Fig. 2) by a $\mathrm{NaIO}_{4}$ oxidation, methylation, acetolysis and 2D NMR spectra analysis. ${ }^{21}$

The antitumor activities of compounds $1,11,17,18,19$ and 20 were preliminarily studied according to the method described by Sasaki and co-workers. ${ }^{5 b}$ ICR mice weighing about $20 \mathrm{~g}$ were used for the bioassay. Seven-day-old Sarcoma-180 ascites $\left(0.2 \mathrm{~mL}\right.$, about $5 \times 10^{6}$ cells $)$ were transplanted into the right groins of mice. The test samples, dissolved in distilled water, were injected daily for 10 days starting $24 \mathrm{~h}$ after tumor implantation. At the end of the 12th day, the mice were killed, and the tumors were extirpated and weighted. The results (Table 1), compared to lentinan and cyclophosphamide (CTX) in the parallel test, suggest that compound $\mathbf{1 1}$ and $\mathbf{1 9}$ may be potent antitumor agents. Low tumor inhibition rates of $\mathbf{1 7}$ and $\mathbf{1 8}$ indicate that structurally highly branched oligosaccharides may not be helpful to their bioactivities. A main chain with $\beta-(1 \rightarrow 6)^{22}$ 


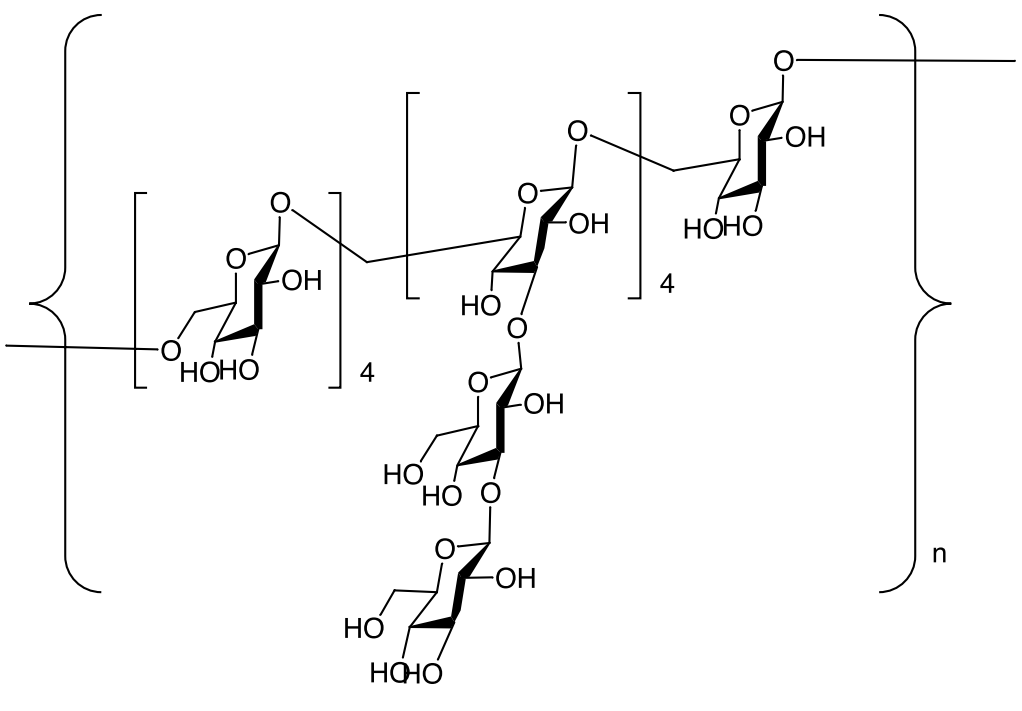

20

Figure 2. Proposed structure of $\beta$-D-glucan isolated from barmy mycelium of Grifola frondosa (Maitake).

Table 1. Preliminary studies on antitumor activities of compounds $\mathbf{1}, \mathbf{1 1}$ and 17-20

\begin{tabular}{|c|c|c|c|c|}
\hline Sample & $\begin{array}{c}\text { Dose } \\
(\mathrm{mg} / \mathrm{Kg})\end{array}$ & $\begin{array}{l}\Delta \text { Body weight } \\
(\mathrm{g})\end{array}$ & $\begin{array}{l}\text { Weight of tumor } \\
\qquad(\mathrm{g})\end{array}$ & $\begin{array}{c}\text { Inhibition rate } \\
(\%)\end{array}$ \\
\hline Control & 0 & $10.4 \pm 2.3$ & $1.42 \pm 0.45$ & 0 \\
\hline CTX & 30 & $8.7 \pm 1.5$ & $0.35 \pm 0.09 * * *$ & 75 \\
\hline Lentinan & 2.0 & $12.9 \pm 2.0$ & $0.95 \pm 0.56^{* *}$ & 33 \\
\hline 1 & 5.0 & $9.6 \pm 1.8$ & $1.15 \pm 0.41 * *$ & 19 \\
\hline 11 & 2.5 & $12.6 \pm 3.2$ & $0.88 \pm 0.46 *$ & 38 \\
\hline 11 & 5.0 & $11.0 \pm 1.1$ & $0.74 \pm 0.23 * *$ & 48 \\
\hline 11 & 10.0 & $10.8 \pm 1.9$ & $0.58 \pm 0.15^{* * *}$ & 59 \\
\hline 17 & 2.0 & $6.9 \pm 1.3$ & $1.11 \pm 0.60^{*}$ & 22 \\
\hline 18 & 2.0 & $9.6 \pm 1.6$ & $1.09 \pm 0.63 *$ & 23 \\
\hline 19 & 1.0 & $12.7 \pm 1.8$ & $0.44 \pm 0.13 * * *$ & 69 \\
\hline 20 & 1.0 & $10.8 \pm 1.3$ & $0.80 \pm 0.22 * *$ & 43 \\
\hline
\end{tabular}

$t$-test: $* p<0.05 ; * * p<0.01 ; * * * p<0.001$.

or $\beta-(1 \rightarrow 3)^{8 b, 9 b}$ linkage can be important. More details about the action mechanism for $\mathbf{1 1}$ and $\mathbf{1 9}$ are currently under investigation by our collaborators.

\section{Conclusions}

A highly efficient and practical method was described for the preparation of 3,6-branched hexa- $\beta$-D-glucopyranosyl derivatives. A dendritic nonasaccharide was also synthesized. The antitumor activities of oligosaccharide $\mathbf{1}$, dendrimer 17, their sulfated derivatives $\mathbf{1 1}$ and 18, together with a natural glucan-protein 19 and the corresponding glucan 20, isolated from barmy mycelium of Grifola frondosa (Maitake), were preliminarily investigated in vivo based on Sarcoma-180 model studies. Our current research suggests that the sulfated branching oligosaccharide and natural glycoprotein have better antitumor activities comparing to the parent sugar residue alone (oligosaccharide or polysaccharide). Beside on this result, some structural closely related glycopeptides and BSA attached glycoconjugates are now under preparation in our lab.

\section{Experimental}

\subsection{General methods}

Optical rotations were determined at $25{ }^{\circ} \mathrm{C}$ with a PerkinElmer Model 241-Mc automatic polarimeter. ${ }^{1} \mathrm{H}$ NMR, ${ }^{13} \mathrm{C}$ NMR and ${ }^{1} \mathrm{H}-{ }^{1} \mathrm{H}$ COSY, NOESY and ${ }^{1} \mathrm{H}-{ }^{13} \mathrm{C}$ COSY spectra were recorded with Bruker ARX 400 or 500 spectrometers in $\mathrm{CDCl}_{3}, \mathrm{CD}_{3} \mathrm{OD}$ or $\mathrm{D}_{2} \mathrm{O}$. Chemical shifts are given in ppm downfield from internal $\mathrm{Me}_{4} \mathrm{Si}$. Mass spectra were measured using MALDITOF-MS with CCA as matrix or recorded with a VG PLATFORM mass spectrometer using the ESI technique to introduce the sample. Thin-layer chromatography (TLC) was performed on silica gel $\mathrm{HF}_{254}$ with detection by charring with $30 \%$ (v/v) $\mathrm{H}_{2} \mathrm{SO}_{4}$ in $\mathrm{MeOH}$ or in some cases by a UV detector. General column chromatography was conducted by elution of a column $(8 \times 200 \mathrm{~mm}, 15 \times 300 \mathrm{~mm}, 35 \times 400 \mathrm{~mm})$ of silica gel (100-200 mesh) with EtOAc-petroleum ether $\left(60-90^{\circ} \mathrm{C}\right)$ as the eluent, while the sulfated products were purified on Sephadex LH-20 column using water as eluent. Solutions were concentrated at $<60{ }^{\circ} \mathrm{C}$ under reduced pressure.

4.1.1. Methyl 2,3,4,6-tetra- $O$-acetyl- $\beta$-D-glucopyranosyl$(1 \rightarrow 6)$-[2,3,4,6-tetra- $O$ - acetyl- $\beta$-D-glucopyranosyl$(1 \rightarrow 3)]$-2,4-di- $O$-acetyl- $\alpha$-D-glucopyranoside (5). To a cooled solution $\left(0^{\circ} \mathrm{C}\right)$ of $3(1.1 \mathrm{~g}, 3.1 \mathrm{mmol})$ and $4(3.2 \mathrm{~g}$, $6.5 \mathrm{mmol})$ in anhydrous $\mathrm{CH}_{2} \mathrm{Cl}_{2}(20 \mathrm{~mL})$ was added TMSOTf $(50 \mu \mathrm{L}, 0.28 \mathrm{mmol})$. The mixture was stirred at these conditions for $4 \mathrm{~h}$ and quenched with $\mathrm{Et}_{3} \mathrm{~N}$. The solvents were evaporated in vacuo and the residue was purified on a silica gel column (petroleum ether-EtOAc, $1: 1)$ to give latent trisaccharide donor $\mathbf{5}$ as a syrup (2.54 g, $82 \%) ;[\alpha]_{\mathrm{D}}^{25}=-11\left(\right.$ c $\left.1, \mathrm{CHCl}_{3}\right) ;{ }^{1} \mathrm{H}$ NMR $(400 \mathrm{MHz}$, $\left.\mathrm{CDCl}_{3}\right) \delta 1.96,1.97,2.00,2.01,2.02,2.03,2.10,2.17,2.21$, $2.23\left(10 \mathrm{~s}, 10 \times 3 \mathrm{H}, \mathrm{COCH}_{3}\right), 3.57$ (ddd, $1 \mathrm{H}, J=5.5,11.9$, $8.8 \mathrm{~Hz}), 3.60-3.66(\mathrm{~m}, 3 \mathrm{H}), 3.78(\mathrm{t}, 1 \mathrm{H}, J=8.8 \mathrm{~Hz}), 4.02$ (dd, $1 \mathrm{H}, J=5.5,11.9 \mathrm{~Hz}), 4.11-4.17(\mathrm{~m}, 2 \mathrm{H}), 4.43(\mathrm{~d}, 1 \mathrm{H}$, $\left.J=7.9 \mathrm{~Hz}, \mathrm{H}-1^{\mathrm{II}}\right), 4.52\left(\mathrm{~d}, 1 \mathrm{H}, J=8.0 \mathrm{~Hz}, \mathrm{H}-1^{\mathrm{III}}\right), 4.54$ (d, $\left.1 \mathrm{H}, J=10.0 \mathrm{~Hz}, \mathrm{H}-1^{\mathrm{I}}\right), 4.57(\mathrm{dd}, 1 \mathrm{H}, J=2.0,11.9 \mathrm{~Hz}), 4.62$ 
(dd, $1 \mathrm{H}, J=3.3,12.5 \mathrm{~Hz}), 4.72(\mathrm{dd}, 1 \mathrm{H}, J=3.3,12.6 \mathrm{~Hz})$, $4.96\left(\mathrm{dd}, 1 \mathrm{H}, J=10.0,10.8 \mathrm{~Hz}, \mathrm{H}-2^{\mathrm{I}}\right), 5.00-5.05(\mathrm{~m}, 2 \mathrm{H})$, 5.11-5.21 (m, 4H), 7.25-7.50 (m, 4H, Ph). Anal. Calcd for $\mathrm{C}_{44} \mathrm{H}_{56} \mathrm{O}_{25} \mathrm{~S}$ : C, 51.97; H, 5.55. Found: C, 52.20; H, 5.48.

4.1.2. Methyl 6-O-tert-butyldimethylsilyl-2,3,4-tri- $O$ acetyl- $\beta$-D-glucopyranosyl- $(1 \rightarrow 6)$-[2,3,4,6-tetra- $O$ acetyl- $\beta$-D-glucopyranosyl- $(1 \rightarrow 3)]$-2,4-di- $O$-acetyl- $\alpha$-Dglucopyranoside $(8)$. To a cold solution $\left(-42{ }^{\circ} \mathrm{C}\right)$ of 6 $(1.37 \mathrm{~g}, 4.91 \mathrm{mmol})$ and $7(2.78 \mathrm{~g}, 4.93 \mathrm{mmol})$ in anhydrous $\mathrm{CH}_{2} \mathrm{Cl}_{2}(20 \mathrm{~mL})$ was added TMSOTf $(60 \mu \mathrm{L}, 0.33 \mathrm{mmol})$. The mixture was stirred at this temperature (usually $2 \mathrm{~h}$ ) until all starting materials were consumed according to TLC (petroleum ether/EtOAc 1/1), and then warmed to $0{ }^{\circ} \mathrm{C}$. Compound 4 (2.42 g, $4.93 \mathrm{mmol})$ in dry $\mathrm{CH}_{2} \mathrm{Cl}_{2}(5 \mathrm{~mL})$ was added into the above mixture dropwise at $0{ }^{\circ} \mathrm{C}$, followed by the addition of extra TMSOTf $(10 \mu \mathrm{L}, 0.05 \mathrm{mmol})$, and the mixture was kept at these conditions for $2 \mathrm{~h}$, then quenched with $\mathrm{Et}_{3} \mathrm{~N}$. The solvents were evaporated in vacuo and the residue was purified by silica gel column chromatography (petroleum ether-EtOAc, 1:1) to give trisaccharide $\mathbf{8}$ as a syrup $(3.77 \mathrm{~g}, 76 \%) ;[\alpha]_{\mathrm{D}}^{25}=+41^{\circ}\left(c 1, \mathrm{CHCl}_{3}\right) ;{ }^{1} \mathrm{H}$ NMR $\left(400 \mathrm{MHz}, \mathrm{CDCl}_{3}\right) \delta 0.03,0.04\left(2 \mathrm{~s}, 6 \mathrm{H},\left(\mathrm{CH}_{3}\right)_{2} \mathrm{Si}\right), 0.88(\mathrm{~s}$, $9 \mathrm{H}, t$-Bu $), 1.97,1.98,1.99,2.01,2.02,2.03,2.07,2.18(8 \mathrm{~s}$, $27 \mathrm{H}, 9 \mathrm{CH}_{3} \mathrm{CO}$ ), 3.38 (s, $\left.3 \mathrm{H}, \mathrm{OCH}_{3}\right), 3.45$ (dd, $1 \mathrm{H}, J=6.8$, $\left.10.7 \mathrm{~Hz}, \mathrm{H}-6 \mathrm{a}^{\mathrm{III}}\right), 3.52\left(\mathrm{ddd}, 1 \mathrm{H}, J=2.9,4.7,9.9 \mathrm{~Hz}, \mathrm{H}-5^{\mathrm{I}}\right)$, 3.64 (ddd, $\left.1 \mathrm{H}, J=2.2,9.4,4.6 \mathrm{~Hz}, \mathrm{H}-5^{\mathrm{II}}\right), 3.66-3.75(\mathrm{~m}, 2 \mathrm{H}$, H-6 ${ }^{\mathrm{I}}$ ), 3.88 (ddd, $\left.1 \mathrm{H}, J=1.8,6.8,9.5 \mathrm{~Hz}, \mathrm{H}-5^{\mathrm{III}}\right), 3.93$ (dd, $1 \mathrm{H}, J=1.8,10.7 \mathrm{~Hz}, \mathrm{H}-6 \mathrm{~b}^{\mathrm{III}}$ ), 4.04 (dd, $1 \mathrm{H}, J=2.2,12.4 \mathrm{~Hz}$, $\left.\mathrm{H}-6 \mathrm{a}^{\mathrm{II}}\right), 4.11\left(\mathrm{t}, 1 \mathrm{H}, J=9.3 \mathrm{~Hz}, \mathrm{H}-3^{\mathrm{I}}\right), 4.34(\mathrm{dd}, 1 \mathrm{H}, J=4.6$, $\left.12.4 \mathrm{~Hz}, \mathrm{H}-6 \mathrm{~b}^{\mathrm{II}}\right), 4.49$ (d, $\left.1 \mathrm{H}, J=8.0 \mathrm{~Hz}, \mathrm{H}-1^{\mathrm{III}}\right), 4.65$ (d, $\left.1 \mathrm{H}, J=8.1 \mathrm{~Hz}, \mathrm{H}-1^{\mathrm{II}}\right), 4.80\left(\mathrm{dd}, 1 \mathrm{H}, J=9.3,9.9 \mathrm{~Hz}, \mathrm{H}-4^{\mathrm{I}}\right)$, $4.81\left(\mathrm{~d}, 1 \mathrm{H}, J=3.7 \mathrm{~Hz}, \mathrm{H}-1^{\mathrm{I}}\right), 4.83(\mathrm{dd}, 1 \mathrm{H}, J=3,7,9.3 \mathrm{~Hz}$, $\left.\mathrm{H}-2^{\mathrm{I}}\right), 4.88\left(\mathrm{dd}, 1 \mathrm{H}, J=8.1,9.3 \mathrm{~Hz}, \mathrm{H}-2^{\mathrm{II}}\right), 4.97(\mathrm{dd}, 1 \mathrm{H}$, $\left.J=8.0,9.5 \mathrm{~Hz}, \mathrm{H}-2^{\mathrm{III}}\right), 5.01\left(\mathrm{t}, 1 \mathrm{H}, J=9.5 \mathrm{~Hz}, \mathrm{H}-4^{\mathrm{III}}\right), 5.04$ $\left(\mathrm{t}, 1 \mathrm{H}, J=9.4 \mathrm{~Hz}, \mathrm{H}-4^{\mathrm{II}}\right), 5.11\left(\mathrm{t}, 1 \mathrm{H}, J=9.4 \mathrm{~Hz}, \mathrm{H}-3^{\mathrm{II}}\right)$, $5.20\left(\mathrm{t}, 1 \mathrm{H}, J=9.5 \mathrm{~Hz}, \mathrm{H}-3^{\mathrm{III}}\right)$. MALDITOF-MS calcd for $\mathrm{C}_{43} \mathrm{H}_{66} \mathrm{O}_{25} \mathrm{Si}: 1010[\mathrm{M}]^{+}$. Found $1033[\mathrm{M}+\mathrm{Na}]^{+}$. Anal. Calcd for $\mathrm{C}_{43} \mathrm{H}_{66} \mathrm{O}_{25} \mathrm{Si}$ : C, 51.08; H, 6.58. Found: C, 51.27; $\mathrm{H}, 6.52$.

4.1.3. Methyl 2,3,4,6-tetra- $O$-acetyl- $\beta$-D-glucopyranosyl$(1 \rightarrow 6)$ - $[2,3,4,6$-tetra- $O$-acetyl- $\beta$-D-glucopyranosyl$(1 \rightarrow 3)]-2,4-d i-O$-acetyl- $\beta$-D-glucopyranosyl- $(1 \rightarrow 6)-2,3,4-$ tri- $O$-acetyl- $\beta$-D-glucopyranosyl- $(1 \rightarrow 6)-[2,3,4,6$-tetra- $O$ acetyl- $\beta$-D-glucopyranosyl-( $1 \longrightarrow 3)]$-2,4-di- $O$-acetyl- $\alpha$-Dglucopyranoside (10). Compound $\mathbf{8}$ (4.55 g, $4.5 \mathrm{mmol}$ ) was stirred in $95 \%$ TFA $(30 \mathrm{~mL})$ at $\mathrm{rt}$ for $1 \mathrm{~h}$ and then evaporated with toluene $(3 \times 50 \mathrm{~mL})$ for 3 times to give the dried crude 9. To a cooled solution $\left(0{ }^{\circ} \mathrm{C}\right)$ of $\mathbf{5}(4.576 \mathrm{~g}, 4.5 \mathrm{mmol})$ and crude 9 (4.03 g, $4.5 \mathrm{mmol})$ in anhydrous $\mathrm{CH}_{2} \mathrm{Cl}_{2}(50 \mathrm{~mL})$ was added TMSOTf ( $60 \mu \mathrm{L}, 0.33 \mathrm{mmol})$. The mixture was stirred at this temperature for $2 \mathrm{~h}$, and then quenched with $\mathrm{Et}_{3} \mathrm{~N}$. The solvents were evaporated in vacuo and the residue was purified by silica gel column chromatography (petroleum ether-EtOAc, 1.5:1) to give hexasaccharide $\mathbf{1 0}$ as a syrup $(6.98 \mathrm{~g}, 86 \%) ;[\alpha]_{\mathrm{D}}^{25}=-3\left(c 4, \mathrm{CHCl}_{3}\right) ;{ }^{1} \mathrm{H} \mathrm{NMR}$ $\left(500 \mathrm{MHz}, \mathrm{CDCl}_{3}\right) \delta 1.93,1.94,1.95,1.96,1.97,1.98,1.99$, $2.00,2.01,2.03,2.05,2.06,2.13,2.15(14 \mathrm{~s}, 57 \mathrm{H}, 19$ $\left.\mathrm{CH}_{3} \mathrm{CO}\right), 3.35\left(\mathrm{~s}, 3 \mathrm{H}, \mathrm{OCH}_{3}\right), 3.42-3.48\left(\mathrm{~m}, 2 \mathrm{H}, \mathrm{H}-5^{\mathrm{I}}\right.$, H-6a $\left.{ }^{\mathrm{I}}\right), 3.50\left(\mathrm{dd}, 1 \mathrm{H}, J=7.0,10.5 \mathrm{~Hz}, \mathrm{H}-6 \mathrm{a}^{\mathrm{II}}\right), 3.54-3.60$ $\left(\mathrm{m}, 2 \mathrm{H}, \mathrm{H}-5^{\mathrm{II}}, \mathrm{H}-5^{\mathrm{III}}\right), 3.62-3.70\left(\mathrm{~m}, 3 \mathrm{H}, \mathrm{H}-5^{\mathrm{IV}}, \mathrm{H}-5^{\mathrm{V}}\right.$, $\left.\mathrm{H}-5^{\mathrm{VI}}\right), 3.79-3.88$ (m, 4H, H-3 $\left.{ }^{\mathrm{III}}, \mathrm{H}-6 \mathrm{~b}^{\mathrm{I}}, \mathrm{H}-6 \mathrm{a}^{\mathrm{III}}, \mathrm{H}-6 \mathrm{~b}^{\mathrm{III}}\right)$, $3.91\left(\mathrm{dd}, 1 \mathrm{H}, J=2.5,10.5 \mathrm{~Hz}, \mathrm{H}-6 \mathrm{~b}^{\mathrm{II}}\right), 4.01$ (dd, $1 \mathrm{H}, J=2.5$, $7.5 \mathrm{~Hz}, \mathrm{H}-6 \mathrm{a}^{\mathrm{VI}}$ ), 4.03 (dd, $\left.1 \mathrm{H}, J=2.0,7.5 \mathrm{~Hz}, \mathrm{H}-6 \mathrm{a}^{\mathrm{V}}\right), 4.06-$ $4.12\left(\mathrm{~m}, 2 \mathrm{H}, \mathrm{H}-6 \mathrm{~b}^{\mathrm{V}}, \mathrm{H}-3^{\mathrm{I}}\right), 4.24(\mathrm{dd}, 1 \mathrm{H}, J=4.5,12.0 \mathrm{~Hz}$, $\left.\mathrm{H}-6 \mathrm{a}^{\mathrm{IV}}\right), 4.29\left(\mathrm{~d}, 1 \mathrm{H}, J=8.0 \mathrm{~Hz}, \mathrm{H}-1^{\mathrm{III}}\right), 4.30(\mathrm{dd}, 1 \mathrm{H}$, $\left.J=3.0,7.5 \mathrm{~Hz}, \mathrm{H}-6 \mathrm{~b}^{\mathrm{VI}}\right), 4.34(\mathrm{dd}, 1 \mathrm{H}, J=4.0,12.0 \mathrm{~Hz}$, $\left.\mathrm{H}-6 \mathrm{~b}^{\mathrm{IV}}\right), 4.49\left(\mathrm{~d}, 1 \mathrm{H}, J=8.0 \mathrm{~Hz}, \mathrm{H}-1^{\mathrm{II}}\right), 4.51(\mathrm{~d}, 1 \mathrm{H}, J=$ $\left.8.0 \mathrm{~Hz}, \mathrm{H}-1^{\mathrm{IV}}\right), 4.58\left(\mathrm{~d}, 1 \mathrm{H}, J=8.0 \mathrm{~Hz}, \mathrm{H}-1^{\mathrm{VI}}\right), 4.61(\mathrm{~d}, 1 \mathrm{H}$, $\left.J=8.0 \mathrm{~Hz}, \mathrm{H}-1^{\mathrm{V}}\right), 4.71\left(\mathrm{t}, 1 \mathrm{H}, J=9.5 \mathrm{~Hz}, \mathrm{H}-4^{\mathrm{III}}\right), 4.77(\mathrm{~d}$, $\left.1 \mathrm{H}, J=3.5 \mathrm{~Hz}, \mathrm{H}-1^{\mathrm{I}}\right), 4.78-4.93(\mathrm{~m}, 7 \mathrm{H}), 4.94(\mathrm{dd}, 1 \mathrm{H}$, $\left.J=8.0,9.5 \mathrm{~Hz}, \mathrm{H}-2^{\mathrm{IV}}\right), 5.01\left(\mathrm{t}, 1 \mathrm{H}, J=9.0 \mathrm{~Hz}, \mathrm{H}-4^{\mathrm{VI}}\right), 5.02$ $\left(\mathrm{t}, 1 \mathrm{H}, J=9.5 \mathrm{~Hz}, \mathrm{H}-4^{\mathrm{V}}\right), 5.03\left(\mathrm{t}, 1 \mathrm{H}, J=9.5 \mathrm{~Hz}, \mathrm{H}-4^{\mathrm{IV}}\right), 5.08$ $\left(\mathrm{t}, 1 \mathrm{H}, J=9.5 \mathrm{~Hz}, \mathrm{H}-3^{\mathrm{VI}}\right), 5.10\left(\mathrm{t}, 1 \mathrm{H}, J=9.0 \mathrm{~Hz}, \mathrm{H}-3^{\mathrm{V}}\right), 5.15$ $\left(\mathrm{t}, 1 \mathrm{H}, J=9.5 \mathrm{~Hz}, \mathrm{H}-3^{\mathrm{II}}\right), 5.18\left(\mathrm{t}, 1 \mathrm{H}, J=9.5 \mathrm{~Hz}, \mathrm{H}-3^{\mathrm{IV}}\right) . \delta_{\mathrm{C}}$ $\left(125 \mathrm{MHz}, \mathrm{CDCl}_{3}\right)$ 20.3, 20.5, 20.6, 20.7, 20.9, 61.7, 61.8, $67.4,68.0,68.1,68.3,68.4,68.5,68.6,68.7,68.9,71.0$, $71.1,71.2,71.6,71.7,71.9,72.5,72.6,72.7,73.0,73.2$, 76.0, 78.7, $96.4\left(\mathrm{C}-1^{\mathrm{I}}\right), \quad 100.6\left(\mathrm{C}-1^{\mathrm{III}}, \mathrm{C}-1^{\mathrm{V}}\right), 100.8$ $\left(\mathrm{C}-1^{\mathrm{VI}}, \mathrm{C}-1^{\mathrm{IV}}\right), 100.9\left(\mathrm{C}-1^{\mathrm{II}}\right), 168.9,169.0,169.3,169.4$, $169.6,169.7,169.8,170.1,170.3,170.4,170.5,170.6$. MALDITOF-MS calcd for $\mathrm{C}_{75} \mathrm{H}_{102} \mathrm{O}_{50}: 1802[\mathrm{M}]^{+}$. Found $1825[\mathrm{M}+\mathrm{Na}]^{+}$. Anal. Calcd for $\mathrm{C}_{75} \mathrm{H}_{102} \mathrm{O}_{50}: \mathrm{C}, 49.95 ; \mathrm{H}$, 5.70. Found: C, 50.21; H, 5.77.H-4H

4.1.4. Methyl $\beta$-D-glucopyranosyl- $(1 \rightarrow 6)-[\beta-D-$ glucopyranosyl- $(1 \rightarrow 3)]-\beta-D-$ glucopyranosyl- $(1 \rightarrow 6)-\beta$-Dglucopyranosyl- $(1 \rightarrow 6)$ - $[\beta$-D-glucopyranosyl- $(1 \rightarrow 3)]-\alpha$ D-glucopyranoside (1). A solution of $\mathbf{1 0}(2.6 \mathrm{~g}, 1.44 \mathrm{mmol})$ in ammonia-saturated $\mathrm{MeOH}(300 \mathrm{~mL})$ was stirred at $\mathrm{rt}$ for 7 days. The solvents were evaporated, and the residue was purified on a Sephadex LH-20 column with water as the eluent to give $\mathbf{1}$ as an amorphous solid after lyophilization $(1.3 \mathrm{~g}, 90 \%) ;[\alpha]_{\mathrm{D}}^{25}=+7\left(c 1, \mathrm{H}_{2} \mathrm{O}\right) ;{ }^{1} \mathrm{H}$ NMR $(500 \mathrm{MHz}$, $\left.\mathrm{D}_{2} \mathrm{O}\right) \delta 3.28(\mathrm{t}, 1 \mathrm{H}, J=9.1 \mathrm{~Hz}),, 3.30(\mathrm{t}, 1 \mathrm{H}, J=9.1 \mathrm{~Hz}), 3.34$ $(\mathrm{t}, 1 \mathrm{H}, J=9.30 \mathrm{~Hz}), 3.37(\mathrm{t}, 1 \mathrm{H}, J=9.5 \mathrm{~Hz}), 3.38-3.48(\mathrm{~m}$, $13 \mathrm{H}), 3.51(\mathrm{t}, 2 \mathrm{H}, J=9.5 \mathrm{~Hz}), 3.58-3.93(\mathrm{~m} 18 \mathrm{H}), 4.15-$ $4.23\left(\mathrm{~m}, 2 \mathrm{H}, \mathrm{H}-3^{\mathrm{I}}, \mathrm{H}-3^{\mathrm{III}}\right), 4.50(\mathrm{~d}, 2 \mathrm{H}, J=7.9 \mathrm{~Hz}), 4.55(\mathrm{~d}$, $1 \mathrm{H}, J=8.0 \mathrm{~Hz}), 4.69(\mathrm{~d}, 1 \mathrm{H}, J=8.0 \mathrm{~Hz}), 4.73(\mathrm{~d}, 1 \mathrm{H}, J=$ $8.0 \mathrm{~Hz}), 4.80(\mathrm{~d}, 1 \mathrm{H}, J=3.7 \mathrm{~Hz}) . \delta_{\mathrm{C}}\left(125 \mathrm{MHz}, \mathrm{CDCl}_{3}\right)$ $55.0,60.5,67.6,67.7,68.4,68.6,69.2,69.3,69.4,70.2$, $70.5,72.6,72.9,73.2,74.4,74.6,75.3,75.4,75.7,75.8$, 82.0, 84.0, 99.0, 102.5, 102.6, 102.7 (3C). ESI-MS calcd for $\mathrm{C}_{37} \mathrm{H}_{64} \mathrm{O}_{31}: 1004[\mathrm{M}]^{+}$, found $1003[\mathrm{M}-\mathrm{H}]^{+}$.

4.1.5. 6-Azidohexyl 2,3,4,6-tetra- $O$-benzoyl- $\beta$-D-glucopyranosyl- $(1 \rightarrow 6)$-[2,3,4,6-tetra- $O$-benzoyl- $\beta$-D-glucopyranosyl-( $1 \longrightarrow 3)]$-2,4-di- $O$-acetyl- $\beta$-D-glucopyranoside (13). To a solution of 2,3,4,6-tetra- $O$-benzoyl- $\beta$-D-glucopyranosyl- $(1 \rightarrow 6)$-[2,3,4,6-tetra- $O$-benzoyl- $\beta$-D-glucopyranosyl- $(1 \rightarrow 3)]$-2,4-di- $O$-acetyl- $\beta$-D-glucopyranosyl trichloroacetimidate $(\mathbf{1 2}, 586 \mathrm{mg}, \quad 0.4 \mathrm{mmol})$ and 6-azido-1hexanol $(52 \mathrm{mg}, 0.4 \mathrm{mmol})$ in anhydrous dichloromethane (3 mL) was added TMSOTf $(10 \mu \mathrm{L}, 0.06 \mathrm{mmol})$ at $0{ }^{\circ} \mathrm{C}$ under $\mathrm{N}_{2}$ protection. The reaction mixture was stirred for $2 \mathrm{~h}$, at the end of which time TLC indicated the completion of the reaction. The mixture was neutralized with $\mathrm{Et}_{3} \mathrm{~N}$, concentrated and purified by flash chromatography using 2:1 petroleum ether-EtOAc as the eluent to give syrupy $\mathbf{1 3}$ (476 mg, 84.7\%); $[\alpha]_{\mathrm{D}}^{20}=-101\left(c 0.5, \mathrm{CHCl}_{3}\right) ;{ }^{1} \mathrm{H}$ NMR $\left(400 \mathrm{~Hz}, \mathrm{CDCl}_{3}\right) \delta 1.06-1.15\left(\mathrm{~m}, 4 \mathrm{H},-\mathrm{CH}_{2} \mathrm{CH}_{2}-\right), 1.19-$ $1.26\left(\mathrm{~m}, 2 \mathrm{H},-\mathrm{CH}_{2} \mathrm{CH}_{2}-\right), 1.45-1.52\left(\mathrm{~m}, 2 \mathrm{H},-\mathrm{CH}_{2} \mathrm{CH}_{2}-\right)$, 1.86 (s, 3H, $\left.\mathrm{CH}_{3} \mathrm{CO}\right), 1.91\left(\mathrm{~s}, 3 \mathrm{H}, \mathrm{CH}_{3} \mathrm{CO}\right), 2.95(\mathrm{~m}, 1 \mathrm{H}$, $\left.\mathrm{OCH}_{2}\right), 3.21\left(\mathrm{t}, 2 \mathrm{H}, \mathrm{CH}_{2} \mathrm{~N}_{3}\right), 3.34-3.38\left(\mathrm{~m}, 1 \mathrm{H}, \mathrm{OCH}_{2}\right)$, $3.49-3.51\left(\mathrm{~m}, 1 \mathrm{H}, \mathrm{H}-5^{\mathrm{I}}\right), 3.62\left(\mathrm{dd}, 1 \mathrm{H}, J_{6 \mathrm{a}, 6 \mathrm{~b}}=11.2 \mathrm{~Hz}\right.$, 
$\left.J_{6 \mathrm{a}, 5}=5.7 \mathrm{~Hz}, \mathrm{H}-6 \mathrm{a}^{\mathrm{I}}\right), 3.78-4.92\left(\mathrm{~m}, 2 \mathrm{H}, \mathrm{H}-3^{\mathrm{I}}, \mathrm{H}-6 \mathrm{~b}^{\mathrm{I}}\right)$, $4.04-4.16$ (m, 3H, H-1 $\left.{ }^{\mathrm{I}}, \mathrm{H}-5^{\mathrm{II}}, \mathrm{H}-5^{\mathrm{III}}\right), 4.41-4.50$ (m, 2H, $2 \mathrm{H}-6), 4.58-4.66$ (m, 2H, 2H-6), 4.69-4.81 (m, 2H, H-2 ${ }^{\mathrm{I}}$ and $\left.\mathrm{H}-4^{\mathrm{I}}\right), 4.89\left(\mathrm{~d}, 1 \mathrm{H}, J_{1,2}=7.6 \mathrm{~Hz}, \mathrm{H}-1\right), 4.91(\mathrm{~d}, 1 \mathrm{H}$, $\left.J_{1,2}=7.8 \mathrm{~Hz}, \mathrm{H}-1\right), 5.36\left(\mathrm{dd}, 1 \mathrm{H}, J_{2,3}=9.6 \mathrm{~Hz}, J_{1,2}=7.8 \mathrm{~Hz}\right.$, H-2), 5.49 (dd, $\left.1 \mathrm{H}, J_{2,3}=9.6 \mathrm{~Hz}, J_{1,2}=7.6 \mathrm{~Hz}, \mathrm{H}-2\right), 5.60-$ $5.68\left(\mathrm{~m}, 2 \mathrm{H}, \mathrm{H}-4^{\mathrm{II}}\right.$ and $\left.\mathrm{H}-4^{\mathrm{III}}\right), 5.83-5.90\left(\mathrm{~m}, 2 \mathrm{H}, \mathrm{H}-3^{\mathrm{II}}\right.$ and H-3 $\left.{ }^{\mathrm{III}}\right), 7.28-8.04(\mathrm{~m}, 40 \mathrm{H}, \mathrm{Ph})$. MALDITOF-MS calcd for $\mathrm{C}_{84} \mathrm{H}_{79} \mathrm{~N}_{3} \mathrm{O}_{26}: 1545[\mathrm{M}]^{+}$, found $1568[\mathrm{M}+\mathrm{Na}]^{+}$. Anal. Calcd for $\mathrm{C}_{84} \mathrm{H}_{79} \mathrm{~N}_{3} \mathrm{O}_{26}$ : C, 65.24; H, 5.15. Found: C, 65.05; H, 5.07 .

4.1.6. 6-Aminohexyl $2,3,4,6$-tetra- $O$-benzoyl- $\beta$-D-glucopyranosyl- $(1 \rightarrow 6)-[2,3,4,6$-tetra- $O$-benzoyl- $\beta$-D-glucopyranosyl- $(1 \rightarrow 3)$ ]-2,4-di- $O$-acetyl- $\beta$-D-glucopyranoside (14). Compound 13 (431 mg, $0.278 \mathrm{mmol}$ ) was dissolved in EtOAc-EtOH $(1: 1,10 \mathrm{~mL})$ containing $\mathrm{Pd}(\mathrm{OH})_{2} / \mathrm{C}(20 \%$, $40 \mathrm{mg}$ ) at rt. $\mathrm{H}_{2}$ was bubbled into the mixture at the flow rate of $100 \mathrm{~mL} / \mathrm{min}$ while stirring at atmospheric pressure for $4 \mathrm{~h}$. The mixture was then filtered over Celite and the filtrate was concentrated under reduced pressure. Purification of the residue by flash chromatography $\left(\mathrm{CH}_{2} \mathrm{Cl}_{2} / \mathrm{MeOH}\right.$ : 5/1) afforded 14 (394 mg, 93.4\%) as a syrup; $[\alpha]_{\mathrm{D}}^{20}=-4(c 1$, $\left.\mathrm{CHCl}_{3}\right) ;{ }^{1} \mathrm{H}$ NMR $\left(400 \mathrm{~Hz}, \mathrm{CDCl}_{3}\right) \delta 1.03-1.14(\mathrm{~m}, 4 \mathrm{H}$, $-\mathrm{CH}_{2} \mathrm{CH}_{2}-$ ), 1.17-1.24 (m, $\left.2 \mathrm{H},-\mathrm{CH}_{2} \mathrm{CH}_{2}-\right)$, 1.61-1.68 (m, $2 \mathrm{H},-\mathrm{CH}_{2} \mathrm{CH}_{2}-$ ), 1.88 (s, 3H, $\left.\mathrm{CH}_{3} \mathrm{CO}\right), 1.89$ (s, 3H, $\left.\mathrm{CH}_{3} \mathrm{CO}\right), 2.86-2.95\left(\mathrm{~m}, 3 \mathrm{H}, \mathrm{CH}_{2} \mathrm{~N}_{3}\right.$ and one proton of $\left.\mathrm{OCH}_{2}\right), 3.34-3.37\left(\mathrm{~m}, 1 \mathrm{H}, \mathrm{OCH}_{2}\right), 3.48-3.51(\mathrm{~m}, 1 \mathrm{H}$, H-5 $\left.{ }^{\mathrm{I}}\right), 3.59\left(\mathrm{dd}, 1 \mathrm{H}, J_{6 \mathrm{a}, 6 \mathrm{~b}}=11.6 \mathrm{~Hz}, J_{6 \mathrm{a}, 5}=5.7 \mathrm{~Hz}, \mathrm{H}-6 \mathrm{a}^{\mathrm{I}}\right)$, $3.85-4.92\left(\mathrm{~m}, 2 \mathrm{H}, \mathrm{H}-3^{\mathrm{I}}, \mathrm{H}-6 \mathrm{~b}^{\mathrm{I}}\right), 4.06\left(\mathrm{~d}, 1 \mathrm{H}, J_{1,2}=7.8 \mathrm{~Hz}\right.$, $\left.\mathrm{H}-1^{\mathrm{I}}\right), 4.07-4.16\left(\mathrm{~m}, 2 \mathrm{H}, \mathrm{H}-5^{\mathrm{II}}\right.$ and $\left.\mathrm{H}-5^{\mathrm{III}}\right), 4.44-4.48(\mathrm{~m}$, $2 \mathrm{H}, \mathrm{H}-6), 4.58-4.65$ (m, 2H, H-6), $4.71-4.76\left(\mathrm{~m}, 2 \mathrm{H}, \mathrm{H}-2^{\mathrm{I}}\right.$ and $\left.\mathrm{H}-4^{\mathrm{I}}\right), 4.91\left(\mathrm{~d}, 1 \mathrm{H}, J_{1,2}=7.8 \mathrm{~Hz}, \mathrm{H}-1\right), 4.92(\mathrm{~d}, 1 \mathrm{H}$, $\left.J_{1,2}=7.6 \mathrm{~Hz}, \mathrm{H}-1\right), 5.36\left(\mathrm{dd}, 1 \mathrm{H}, J_{2,3}=9.6 \mathrm{~Hz}, J_{1,2}=7.8 \mathrm{~Hz}\right.$, $\mathrm{H}-2), 5.50\left(\mathrm{dd}, 1 \mathrm{H}, J_{2,3}=9.6 \mathrm{~Hz}, J_{1,2}=7.6 \mathrm{~Hz}, \mathrm{H}-2\right), 5.62-$ $5.69\left(\mathrm{~m}, 2 \mathrm{H}, \mathrm{H}-4^{\mathrm{II}}\right.$ and $\left.\mathrm{H}-4^{\mathrm{III}}\right), 5.85-5.91\left(\mathrm{~m}, 2 \mathrm{H}, \mathrm{H}-3^{\mathrm{II}}\right.$ and H-3 ${ }^{\mathrm{III}}$ ), 7.25-8.02 (m, 40H, Ph). MALDITOF-MS calcd for $\mathrm{C}_{84} \mathrm{H}_{81} \mathrm{NO}_{26}: 1519[\mathrm{M}]^{+}$, found $1542[\mathrm{M}+\mathrm{Na}]^{+}$. Anal. Calcd for $\mathrm{C}_{84} \mathrm{H}_{81} \mathrm{NO}_{26}$ : C, 66.35; H, 5.37. Found: C, 66.21; $\mathrm{H}, 5.45$.

4.1.7. Fully protected dendrimer 16. A mixture of compound 14 (375 mg, $0.2 \mathrm{mmol})$, the triacid 15 (22 mg, $0.08 \mathrm{mmol})$ and HOBT $(33 \mathrm{mg}, 0.2 \mathrm{mmol})$ in dry DMF $(3 \mathrm{~mL})$ was stirred at $0{ }^{\circ} \mathrm{C}$ for $0.5 \mathrm{~h}$. Then DCC $(51 \mathrm{mg}$, $0.248 \mathrm{mmol}$ ) was added and the reaction mixture was stirred at $0{ }^{\circ} \mathrm{C}$ for $0.5 \mathrm{~h}$, then at $\mathrm{rt}$ for $30 \mathrm{~h}$. The mixture was filtered and the filtrate was concentrated. The resulting crude product was diluted in EtOAc $(30 \mathrm{~mL})$ and subsequently washed successively with $5 \% \mathrm{HCl}$, saturated aqueous $\mathrm{NaHCO}_{3}$ and water. The organic phase was concentrated and purified by flash chromatography (petroleum etherEtOAc, 1:5) to complete $\mathbf{1 6}(219 \mathrm{mg}, 57.2 \%)$ as a syrup; $[\alpha]_{\mathrm{D}}^{20}=-6\left(c 1, \mathrm{CHCl}_{3}\right) ;{ }^{1} \mathrm{H} \mathrm{NMR}\left(400 \mathrm{~Hz}, \mathrm{CDCl}_{3}\right) \delta 1.04-$ $1.18\left(\mathrm{~m}, 3 \times 4 \mathrm{H},-\mathrm{CH}_{2} \mathrm{CH}_{2}-\right), 1.29-1.38(\mathrm{~m}, 3 \times 2 \mathrm{H}$, $\left.-\mathrm{CH}_{2} \mathrm{CH}_{2}-\right), 1.58-1.72\left(\mathrm{~m}, 3 \times 2 \mathrm{H},-\mathrm{CH}_{2} \mathrm{CH}_{2}-\right), 1.86(\mathrm{~s}$, $\left.3 \times 3 \mathrm{H}, \mathrm{CH}_{3} \mathrm{CO}\right), 1.89\left(\mathrm{~s}, 3 \times 3 \mathrm{H}, \mathrm{CH}_{3} \mathrm{CO}\right), 2.04-2.08(\mathrm{~m}$, $\left.3 \times 2 \mathrm{H}, \mathrm{CH}_{2}\right), 2.18-2.23\left(\mathrm{~m}, 3 \times 2 \mathrm{H}, \mathrm{CH}_{2}\right), 2.91-2.98(\mathrm{~m}$, $\left.3 \times 1 \mathrm{H}, \mathrm{OCH}_{2}\right), 3.06-3.13\left(\mathrm{~m}, 3 \times 2 \mathrm{H}, \mathrm{CH}_{2} \mathrm{NHCO}\right), 3.34-$ $3.39\left(\mathrm{~m}, 3 \times 1 \mathrm{H}, \mathrm{OCH}_{2}\right), 3.47-3.51\left(\mathrm{~m}, 3 \times 1 \mathrm{H}, \mathrm{H}-5^{\mathrm{I}}\right), 3.63$ $\left(\mathrm{dd}, 3 \times 1 \mathrm{H}, J_{6 \mathrm{a}, 6 \mathrm{~b}}=11.7 \mathrm{~Hz}, J_{6 \mathrm{a}, 5}=6.8 \mathrm{~Hz}, \mathrm{H}-6 \mathrm{a}^{\mathrm{I}}\right), 3.81-4.90$ $\left(\mathrm{m}, 3 \times 2 \mathrm{H}, \mathrm{H}-3^{\mathrm{I}}\right.$ and $\left.\mathrm{H}-6 \mathrm{~b}^{\mathrm{I}}\right), 4.05-4.15\left(\mathrm{~m}, 3 \times 3 \mathrm{H}, \mathrm{H}-1^{\mathrm{I}}\right.$, $\mathrm{H}-5^{\mathrm{II}}$ and $\left.\mathrm{H}-5^{\mathrm{III}}\right), 4.42-4.49$ (m, $\left.3 \times 2 \mathrm{H}, \mathrm{H}-6\right), 4.59-4.65(\mathrm{~m}$,
$3 \times 2 \mathrm{H}, \mathrm{H}-6), 4.70-4.79\left(\mathrm{~m}, 3 \times 2 \mathrm{H}, \mathrm{H}-2^{\mathrm{I}}\right.$ and $\left.\mathrm{H}-4^{\mathrm{I}}\right), 4.90(\mathrm{~d}$, $\left.3 \times 1 \mathrm{H}, J_{1,2}=7.8 \mathrm{~Hz}, \mathrm{H}-1\right), 4.92\left(\mathrm{~d}, 3 \times 1 \mathrm{H}, J_{1,2}=7.6 \mathrm{~Hz}, \mathrm{H}-1\right)$, $5.36\left(\mathrm{dd}, 3 \times 1 \mathrm{H}, J_{2,3}=9.6 \mathrm{~Hz}, J_{1,2}=7.8 \mathrm{~Hz}, \mathrm{H}-2\right), 5.50(\mathrm{dd}$, $\left.3 \times 1 \mathrm{H}, \quad J_{2,3}=9.6 \mathrm{~Hz}, \quad J_{1,2}=7.6 \mathrm{~Hz}, \mathrm{H}-2\right), 5.60-5.68(\mathrm{~m}$, $3 \times 2 \mathrm{H}, \mathrm{H}-4^{\mathrm{II}}$ and $\left.\mathrm{H}-4^{\mathrm{III}}\right), 5.84-5.91\left(\mathrm{~m}, 3 \times 2 \mathrm{H}, \mathrm{H}-3^{\mathrm{II}}\right.$ and $\mathrm{H}-3^{\mathrm{III}}$ ), 6.04 (br t, $\left.3 \times 1 \mathrm{H}, J=5.5 \mathrm{~Hz}, \mathrm{NH}\right), 7.25-8.02$ (m, $3 \times 40 \mathrm{H}, P h)$. MALDITOF-MS calcd for $\mathrm{C}_{262} \mathrm{H}_{252} \mathrm{~N}_{4} \mathrm{O}_{83}$ : $4781[\mathrm{M}]^{+}$, found: $4804[\mathrm{M}+\mathrm{Na}]^{+}$.

4.1.8. Free dendrimer 17. To a solution of $\mathbf{1 6}(196 \mathrm{mg}$, $0.04 \mathrm{mmol})$ in $\mathrm{MeOH}(5 \mathrm{~mL})$ was added $\mathrm{NaOMe}$ until the $\mathrm{pH}$ reached 10 . The mixture was stirred at $\mathrm{rt}$ for 2 days, neutralized with Amberlite IR-120 $\left(\mathrm{H}^{+}\right)$. The solvents were filtered, and the filtrate was concentrated to dryness under reduced pressure. The residue was subjected to chromatography on a Sephadex LH-20 column with $\mathrm{MeOH}$ as the eluent to give $\mathbf{1 7}$ as a white solid $(76 \mathrm{mg}, 91.5 \%) ;[\alpha]_{\mathrm{D}}^{20}=-5$ (c $\left.1, \mathrm{CH}_{3} \mathrm{OH}\right) ;{ }^{1} \mathrm{H}$ NMR (400 Hz, $\left.\mathrm{CD}_{3} \mathrm{OD}\right) \delta 1.19-1.36(\mathrm{~m}$, $\left.3 \times 4 \mathrm{H},-\mathrm{CH}_{2} \mathrm{CH}_{2}-\right), 1.48-1.56\left(\mathrm{~m}, 3 \times 2 \mathrm{H},-\mathrm{CH}_{2} \mathrm{CH}_{2}-\right)$, $1.58-1.66\left(\mathrm{~m}, 3 \times 2 \mathrm{H},-\mathrm{CH}_{2} \mathrm{CH}_{2}-\right), 2.06-2.18(\mathrm{~m}, 3 \times 4 \mathrm{H}$, $\left.-\mathrm{CH}_{2} \mathrm{CH}_{2}-\right), 3.07$ (t, $\left.3 \times 2 \mathrm{H}, \mathrm{CH}_{2} \mathrm{NHCO}\right), 3.12(\mathrm{dd}, 3 \times 1 \mathrm{H}$, $\left.J_{2,3}=9.6 \mathrm{~Hz}, J_{3,4}=10.2 \mathrm{~Hz}, \mathrm{H}-3^{\mathrm{I}}\right), 3.14-3.60(\mathrm{~m}, 3 \times 16 \mathrm{H})$, $3.71\left(\mathrm{dd}, 3 \times 1 \mathrm{H}, J_{6 \mathrm{a}, 6 \mathrm{~b}}=11.5 \mathrm{~Hz}, J_{6 \mathrm{a}, 5}=5.5 \mathrm{~Hz}, \mathrm{H}-6\right), 3.76-$ $3.83(\mathrm{~m}, 3 \times 2 \mathrm{H}), 4.04-4.09(\mathrm{~m}, 3 \times 1 \mathrm{H}), 4.23(\mathrm{~d}, 3 \times 1 \mathrm{H}$, $\left.J_{1,2}=7.8 \mathrm{~Hz}, \mathrm{H}-1\right), 4.31\left(\mathrm{~d}, 3 \times 1 \mathrm{H}, J_{1,2}=7.6 \mathrm{~Hz}, \mathrm{H}-1\right), 4.47$ (d, $\left.3 \times 1 \mathrm{H}, J_{1,2}=7.6 \mathrm{~Hz}, \mathrm{H}-1\right) ; \delta_{\mathrm{C}}\left(100 \mathrm{~Hz}, \mathrm{CD}_{3} \mathrm{OD}\right) 26.7$, 30.2, 30.6, 31.2, $40.5\left(\mathrm{CH}_{2} \mathrm{NHCO}\right), 62.6,62.8,69.8,70.0$, 71.0, 71.60, 74.4, 75.1, 75.5, 76.8, 77.8, 78.0, 78.2, 94.4 $\left(C \mathrm{NO}_{2}\right), 103.9(C-1), 105.0(C-1), 105.2(C-1), 174.0$ (NHCO). MALDITOF-MS Calcd for $\mathrm{C}_{82} \mathrm{H}_{144} \mathrm{~N}_{4} \mathrm{O}_{53}$ : 2032 $[\mathrm{M}]^{+}$, found: $2055[\mathrm{M}+\mathrm{Na}]^{+} ; 2071[\mathrm{M}+\mathrm{K}]^{+}$.

4.1.9. General procedure for sulfation of compounds 1 and 17. A mixture of oligosaccharide $(\mathbf{1}$ or 17$)$ and $\mathrm{SO}_{3} \cdot \mathrm{Pyr}$ (10 equiv.) in DMF was stirred at $50{ }^{\circ} \mathrm{C}$ for 3 days. Before $3 \mathrm{~N} \mathrm{NaOH}$ was added, pyridine was removed in vacuo, and the residue was purified on a Sephadex LH-20 column using water as eluent furnished a mixture of sulfated compound $\mathbf{1 1}$ or $\mathbf{1 8}$ which were used for the next bioassay after freezedrying. Microanalysis data for compound 11: C 16.22\%, H $1.73 \%$, S 19.90\%. Microanalysis for compound 18: C $21.01 \%$, H $2.54 \%$, S $17.79 \%$.

\section{Acknowledgements}

This work was supported by National Basic Research Program of China (2003CB415001) and NNSF of China (Project 30330690, 20372081 and 30370345). The authors would like to thank Professor Jingrong Cui of Peking University and Dr. Hongyan Hu from General Hospital of AP for performing the mice tests.

\section{References and notes}

1. (a) Suzuki, T.; Tsuzuki, A.; Ohno, N.; Ohshima, Y.; Adachi, Y.; Yadomae, T. Biol. Pharm. Bull. 2002, 25, 140-144. (b) Witczak, Z. J. Curr. Med. Chem. 1995, 1, 392-405. (c) Dubourdieu, D.; Ribereau-Gayon, P. Carbohydr. Res. 1981, 93, 294-299. (d) Kitamura, S.; Hori, T.; Kurita, K.; Takeo, K.; Hara, C.; Itoh, W.; Tabata, K.; Elgsaeter, A.; 
Stokke, B. T. Carbohydr. Res. 1994, 263, 111-121. For synthesis: (e) Colonna, B.; Harding, V. D.; Nepogodiev, S. A.; Raymo, F. M.; Spencer, N.; Stoddart, J. F. Chem. Eur. J. 1998, 4, 1244-1254. (f) Amaya, T.; Tanaka, H.; Yamaguchi, T.; Shibuya, N.; Takahashi, T. Tetrahedron Lett. 2001, 52, 9191-9194. (g) Takeo, K.; Maki, K.; Wada, Y.; Kitamura, S. Carbohydr. Res. 1993, 245, 81-96. (h) Takeo, K.; Kawaguchi, M.; Kitamura, S. J. Carbohydr. Chem. 1993, 12, 1043-1056. (i) Yang, G.; Kong, F. Synlett 2000, 1423-1426.

2. Tabata, K.; Ito, W.; Kojima, T.; Kawabata, S.; Misaki, A. Carbohydr. Res. 1981, 89, 121-135.

3. Bluhm, T. L.; Deslands, Y.; Marchessault, R. H.; Perez, S.; Rinaudo, M. Carbohydr. Res. 1982, 100, 117-130.

4. Schmid, F.; Stone, B. A.; McDougall, B. M.; Bacic, A.; Martin, K. L.; Brownlee, R. T. C.; Chai, E.; Seviour, R. J. Carbohydr. Res. 2001, 331, 163-171.

5. (a) Chihara, G.; Maeda, Y.; Hamura, J.; Sasaki, T.; Fukuoka, F. Nature 1969, 222, 687-688. (b) Sasaki, T.; Takasuka, N. Carbohydr. Res. 1976, 47, 99-104.

6. Falch, B. H.; Espevik, T.; Ryan, L.; Stokke, B. T. Carbohydr. Res. 2000, 329, 587-596, and the references cited therein.

7. Tsuzuki, A.; Tateishi, T.; Ohno, N.; Adachi, Y.; Yadomae, T. Biosci. Biotechnol. Biochem. 1999, 63, 104-110.

8. (a) He, H.; Yang, F.; Du, Y. Carbohydr. Res. 2002, 337, 1673-1678. (b) He, H.; Gu, G.; Du, Y. J. Carbohydr. Chem. 2003, 22, 275-283. (c) Yang, F.; He, H.; Du, Y.; Lu, M. Carbohydr. Res. 2002, 337, 1165-1169.

9. (a) Parish, C. R.; Freeman, C.; Brown, K. J.; Francis, D. J.; Cowden, W. B. Cancer Res. 1999, 59, 3433-3441. (b) Katsuraya, K.; Nakashima, H.; Yamamoto, N.; Uryu, T. Carbohydr. Res. 1999, 315, 234-242. (c) Koyanagi, S.; Tanigawa, N.; Nakagawa, H.; Soeda, S.; Shimeno, H. Biochem. Pharmacol. 2003, 65, 173-179. (d) Hahnenberger, R.; Jakobson, A. M. Glycoconj. J. 1991, 8, 350-353. (e) Khoo, K.-H.; Sarda, S.; Xu, X.; Caulfield, J. P.; McNeil, M. R.; Homans, S. W.; Morris, H. R.; Dell, A. J. Biol. Chem. 1995, 270, 17114-17123. (f) Soeda, S.; Kozako, T.; Iwata, K.; Shimeno, H. Biochim. Biophys. Acta 2000, 1497, 127-134.
10. (a) Sharp, J. K.; McNeil, M.; Albersheim, P. J. Biol. Chem. 1984, 259, 11321-11336. (b) Aldington, S.; Fry, S. C. Adv. Bot. Res. 1992, 19, 1-101.

11. Yamada, H.; Harada, T.; Takahashi, T. J. Am. Chem. Soc. 1994, 116, 7919-7920.

12. Hong, N.; Ogawa, T. Tetrahedron Lett. 1990, 31, 3179-3182.

13. (a) Du, Y.; Zhang, M.; Kong, F. Org. Lett. 2000, 2, 3797-3800. (b) Du, Y.; Zhang, M.; Kong, F. Tetrahedron 2001, 57, 1757-1763.

14. Schmidt, R. R.; Michel, J. J. Carbohydr. Chem. 1985, 4, $141-169$.

15. Du, Y.; Gu, G.; Wei, G.; Hua, Y.; Linhardt, R. J. Org. Lett. 2003, 5, 3627-3630.

16. Yu, B.; Xie, J.; Deng, S.; Hui, Y. J. Am. Chem. Soc. 1999, 121, 12196-12197.

17. Auzanneau, F.-I.; Forooghian, F.; Pinto, B. M. Carbohydr. Res. 1996, 291, 21-41.

18. (a) Jayaraman, N.; Nepogodiev, S. A.; Stoddart, J. F. Chem. Eur. J. 1997, 3, 1193-1199. (b) Roy, R. Polym. News 1996, 21, 226-232. (c) Kieburg, C.; Lindhorst, T. K. Tetrahedron Lett. 1997, 38, 3885-3888.

19. (a) Spaltenstein, S.; Whitesides, G. M. J. Am. Chem. Soc. 1991, 113, 686-687. (b) Mortell, K. H.; Gingras, M.; Kiessling, L. L. J. Am. Chem. Soc. 1994, 116, 12053-12054. (c) Manning, D. D.; Hu, X.; Beck, P.; Kiessling, L. L. J. Am. Chem. Soc. 1997, 119, 3161-3162. (d) Thoma, G.; Duthaler, R. O.; Magnani, J. L.; Patton, J. T. J. Am. Chem. Soc. 2001, 123, 10113-10114. (e) Kitov, P. I.; Sadowaska, J. M.; Mulvey, G.; Armstrong, G. D.; Ling, H.; Pannu, N. S.; Read, R. J.; Bundle, D. R. Nature 2000, 403, 669-672. (f) Zanini, D.; Roy, R. J. Am. Chem. Soc. 1997, 119, 2088-2095.

20. Newkome, G. R.; Moorrefield, C. N.; Theriot, K. J. J. Org. Chem. 1988, 53, 5552-5554.

21. The details regarding the extraction, purification and structure determination will be published elsewhere.

22. Nanba, H.; Hamaguchi, A.; Kuroda, H. Chem. Pharm. Bull. 1987, 35, 1162-1168. 